\title{
In vitro 3D angiogenesis assay in egg white matrix: comparison to Matrigel, compatibility to various species, and suitability for drug testing
}

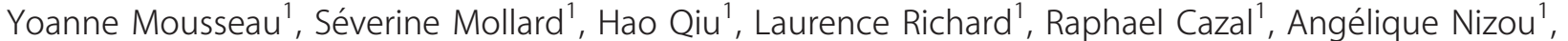 \\ Nicolas Vedrenne ${ }^{1}$, Séverine Rémi ${ }^{2}$, Yasser Baaj ${ }^{1}$, Laurent Fourcade ${ }^{1}$, Benoit Funalot ${ }^{1}$ and Franck G Sturtz ${ }^{1}$
}

In vitro angiogenesis assays are commonly used to assess pro- or anti-angiogenic drug properties. Extracellular matrix (ECM) substitutes such as Matrigel and collagen gel became very popular in in vitro 3D angiogenesis assays as they enable tubule formation by endothelial cells from culture or aortic rings. However, these assays are usually used with a single cell type, lacking the complex cellular interactions occurring during angiogenesis. Here, we report a novel angiogenesis assay using egg white as ECM substitute. We found that, similar to Matrigel, egg white elicited prevascular network formation by endothelial and/or smooth muscle cell coculture. This matrix was suitable for various cells from human, mouse, and rat origin. It is compatible with aortic ring assay and also enables vascular and tumor cell coculture. Through simple labeling (DAPI, Hoechst 33258), cell location and resulting prevascular network formation can easily be quantified. Cell transfection with green fluorescent protein improved whole cell visualization and 3D structure characterization. Finally, eggbased assay dedicated to angiogenesis studies represents a reliable and cost-effective way to produce and analyze data regarding drug effects on vascular cells.

Laboratory Investigation (2014) 94, 340-349; doi:10.1038/labinvest.2013.150; published online 6 January 2014

KEYWORDS: angiogenesis assay; aortic ring; egg white; endothelial cell; smooth muscle cell

With regard to the limitations of 2D cell culture and in order to provide reliable alternative to animal testing, a variety of in vitro $3 \mathrm{D}$ assays have been developed to test angiogenesis inducers and inhibitors. Such assays also provide means to study vascular cell communications, adhesion, and spatial organization following various approaches. In the case of endothelial and mural cell coculture, vascular smooth muscle cells (VSMCs) secrete basement membrane proteins that enable adhesion of endothelial cells (ECs). ${ }^{1-4}$ In addition, proangiogenic factors released by VSMCs promote development and stabilization of capillaries. VSMCs and ECs grow in a three-dimensional (3D) multilayered structure similar to vascular wall structure. Recently, a method using a paper platform for 3D cell culture has been developed. ${ }^{5}$ With this method, cell distribution, growth, and migration can be assessed taking into account the differential oxygen and nutrient diffusion among the different cell layers. ${ }^{6}$ Other methods use substitutes of extracellular matrix (ECM) such as fibrin, collagen, or Matrigel to induce capillary-like formation principally from ECs. ${ }^{7-11}$ These matrices are commercially available from a variety of providers, enabling a highthroughput screening in multiwell plates. In such matrix, ECs grow and migrate as a network representing a preliminary step of neocapillary formation.

At the interface between in vitro and in vivo models, organotypic assays such as aortic ring implantation in an ECM enable tubule formation from a preexisting vascular wall. ${ }^{12}$ Finally, neoangiogenesis can be assessed with the in vivo/ in vitro chick chorioallantoic membrane (CAM) assay, ${ }^{13}$ the in vivo Matrigel plug, the zebrafish, and the corneal neovascularization assays. ${ }^{14}$

In vitro tests are valuable, can be carried out expeditiously, and lend themselves to quantification. ECM based-assays are most of the time used to study a single cell population, whereas angiogenesis implies different cell type communication and interactions. In vivo tests are more difficult and

'Department of Biochemistry and Molecular Genetics, EA 6063, CHU Dupuytren, Limoges, France and ${ }^{2}$ INSERM U 643, Nantes, France

Correspondence: Professor FG Sturtz, MD, PhD, EA 6309, Laboratory of Biochemistry, School of Medicine, University of Limoges, 2 Rue du Dr Raymond Marcland, Limoges F-87025, France.

E-mail: franck.sturtz@unilim.fr

Received 8 April 2013; accepted 23 October 2013 
time consuming to perform, thereby limiting the number of tests that can run at any one time. Quantification is also generally more difficult. As a consequence, angiogenesis assays need to be reliable, cost effective, adapted to cell imaging and labeling, and enable cell interactions studies.

Egg white was recently described as a good alternative of Matrigel for 3D organotypic culture of mammary gland cells. ${ }^{15}$ In order to develop a cost-effective and multipurpose angiogenesis assay, we checked whether egg white could constitute a proper substitute of ECM for vascular cell culture. We used cells from human and rat origin to test their growth and migration abilities in such a matrix. We intended to evaluate the feasibility of 'prevascular' network observation during long periods of time as well as observation and quantification of cell organization in three dimensions.

\section{MATERIALS AND METHODS \\ Cell Culture and Chemicals}

Epirubicin, sunitinib malate (SU11248), and fingolimod (FTY720) were purchased from LC Laboratories (Woburn, MA, USA). Human umbilical vein endothelial cells (HUVECs) and human umbilical artery smooth muscle cells (HASMCs) were obtained by enzymatic digestion of umbilical vessels. ${ }^{16}$ Rat aortic endothelial cells (RAECs) and smooth muscle cells (RASMCs) were obtained from thoracic aorta explants from 3-week-old male Sprague-Dawley rats. ${ }^{17,18}$ Green fluorescent RAECs and RASMCs were obtained with the same methods from GFP-expressing rats kindly given by Ignacio Anegon (INSERM U 643, Nantes, France). After three passages, cells were characterized as endothelial or smooth muscle cells by immunostaining with antibodies directed against Von Willebrand factor (Abcam, Cambridge, UK) or $\alpha$-smooth muscle actin (Sigma-Aldrich, St Louis, MI, USA). Primary cultures of HUVECs and RAECs were used between passages three and eight and cultured in M199 medium (Life Technologies, Carlsbad, CA, USA) supplemented with EC growth factors (ECGFs, Sigma) and 10\% heat-inactivated FBS (BioWhittaker, Walkersville, MD, USA). Primary HASMC and RASMC cultures were used between passages 5 and 15 and were cultured in Dulbecco's modified Eagle medium (DMEM) supplemented with 10\% heatinactivated FBS. These cultures were maintained at $37^{\circ} \mathrm{C}$, under an atmosphere containing $5 \% \mathrm{CO}_{2}$. They were passaged before confluence, and the medium was supplemented twice weekly.

\section{Three-Dimensional Cell Culture}

According to the previously reported method, ${ }^{15}$ egg outer surface was thoroughly washed with ethanol $70 \%$. Under a laminar flow hood, a small hole in the shell was made using sterilized forceps then the egg white was poured into a becher. Egg white was not completely removed from the shell to prevent the egg yolk from mixing with it. The white of 20 eggs was pooled, aliquoted into $15 \mathrm{ml}$ falcon, and stored at $-80{ }^{\circ} \mathrm{C}$ until use. For cell culture, we thawed the egg white to room temperature and we added $100 \mu \mathrm{l}$ of egg white to each well of an 8-well chamber slide or $300 \mu \mathrm{l}$ per well in a 24-well plate using a $1 \mathrm{ml}$ syringe. Both cell culture supports were incubated at $60^{\circ} \mathrm{C}$ in a hybridization oven for $30 \mathrm{~min}$. Once the egg white became semisolid, we seeded the cells in 100 to $500 \mu \mathrm{l}$ of supplemented medium.

\section{Aortic Ring Assay}

After gas anesthesia (2\% isoflurane, Baxter, Maurepas, France), GFP-expressing rats were killed by intracardiac injection of urethane according to good animal handling guidelines. Animal thorax was opened with scissors and thoracic aorta was sampled and washed in PBS containing $1 \%$ penicillin-streptomycin to remove blood. Aorta was then dissected under binocular optics to remove adventice, and cut into $2 \mathrm{~mm}$ pieces that were seeded in egg white matrix.

\section{Cell Labeling and Network Parameter Quantification}

To analyze cell network characteristics, cell nuclei were labeled with $100 \mathrm{ng} / \mathrm{ml}$ Hoechst 33258 (Sigma) that was added $60 \mathrm{~min}$ before the first cell observation. This staining enabled cell nuclei visualization for 2 to 4 days without noticeable induced cell death. Specific cell type identification was carried out after cell fixation with paraformaldehyde $4 \%$ and permeabilization with PBS-Triton X-100 0.3\%. To characterize the cells forming a network as smooth muscle cells, anti$\alpha$-smooth muscle actin antibody was incubated overnight at $4{ }^{\circ} \mathrm{C}$. Cells were rinsed three times with PBS, and incubated with an Alexa 488-conjugated goat anti-mouse antibody (Molecular Probes, Eugene, OR, USA). Image analysis was performed by two minware independent investigators using a Leica DM IRB microscope linked to a CCD camera driven by NIS-AR software.

\section{Confocal Imaging}

Confocal and macroconfocal imaging were respectively performed on a Zeiss LSM510Meta and on a Zeiss AxioZoom V16 equiped with a AxioCam HRm3 S/N 106 camera.

\section{Statistical Analysis}

Statistical significance was determined by Mann-Whitney tests using StatView ${ }^{\circledR} 5.0$ software (SAS Institute, Cary, NC, USA). The minimal level of significance was $P=0.05$.

\section{RESULTS}

\section{Egg White Selection, Use, and Conservation}

We first tested the variety of chicken eggs that are commercially available. Most of the different quality products appeared to be convenient to use as $3 \mathrm{D}$ matrix for angiogenesis assay. However, we rejected the cheapest eggs where the white composition and viscosity appeared too heterogeneous. In fact, the white of this sort of eggs appeared much more liquid than those of higher quality. Consequently, it was more difficult to make the egg white become semi-solid after heating 
to render it a proper matrix for cell culture. We followed a previously described procedure ${ }^{15}$ to extract the white from the eggs. By pooling the white of a large number of eggs simultaneously, we obtained enough egg white to carry out experiments over a long period of time with a homogenous cell culture matrix. The use of the same pool of egg white after more than a year of storage at $-80^{\circ} \mathrm{C}$ did not seem to affect its physical or biological properties. After freezing, egg white has just to be thawed at room temperature and it can be seeded into multiwell chamber slide or plate. Heating time has to be adapted depending on the egg white volume seeded into the wells between 20 and $45 \mathrm{~min}$ for 8 -well chamber slides to 12-well plates, respectively. The use of a hybridization oven seemed to produce more homogeny in the egg white heating compared with the heating block previously described, ${ }^{15}$ enabling the use of egg white matrix in various cell culture devices.

\section{Egg-Based Assay (EBA) Compatibility and Accuracy Versus Matrigel}

Considering that most of angiogenesis assays are carried out on HUVECs, we evaluated the ability of egg white as a multispecies ECM substitute. ECs from humans (HUVECs) and rats (RAECs) appeared to be able to grow, migrate, and organize themselves as 'prevascular' network within $48 \mathrm{~h}$ in such a matrix similar to that observed with Matrigel (Figure 1). After that, the cell network weakly changed for 7 days without medium modification such as growth factor or inhibitor addition. For ECs from humans or rats, network formation speeds were comparable. At $48 \mathrm{~h}$ after cell seeding, the networks were established and stable for up to 1 week if cell medium was replaced every 2 days. Cells associate themselves as a network almost in a plane in Matrigel. Nuclear staining with Hoechst 33258 suggested that their dispersion in egg white seemed to be in a deeper space and revealed a denser
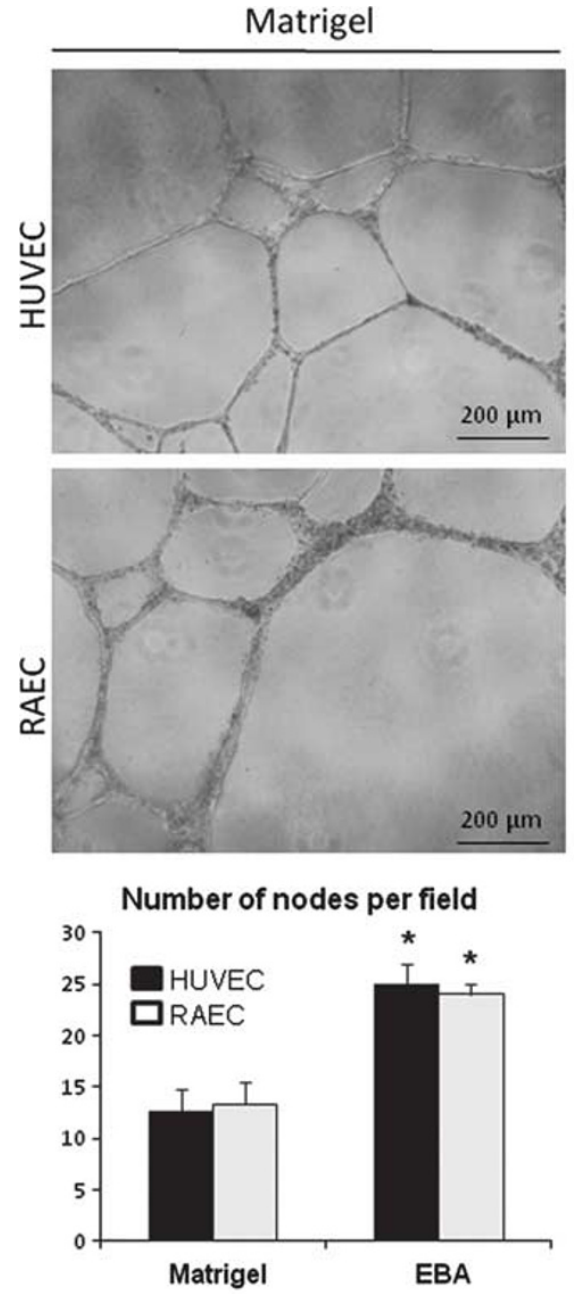
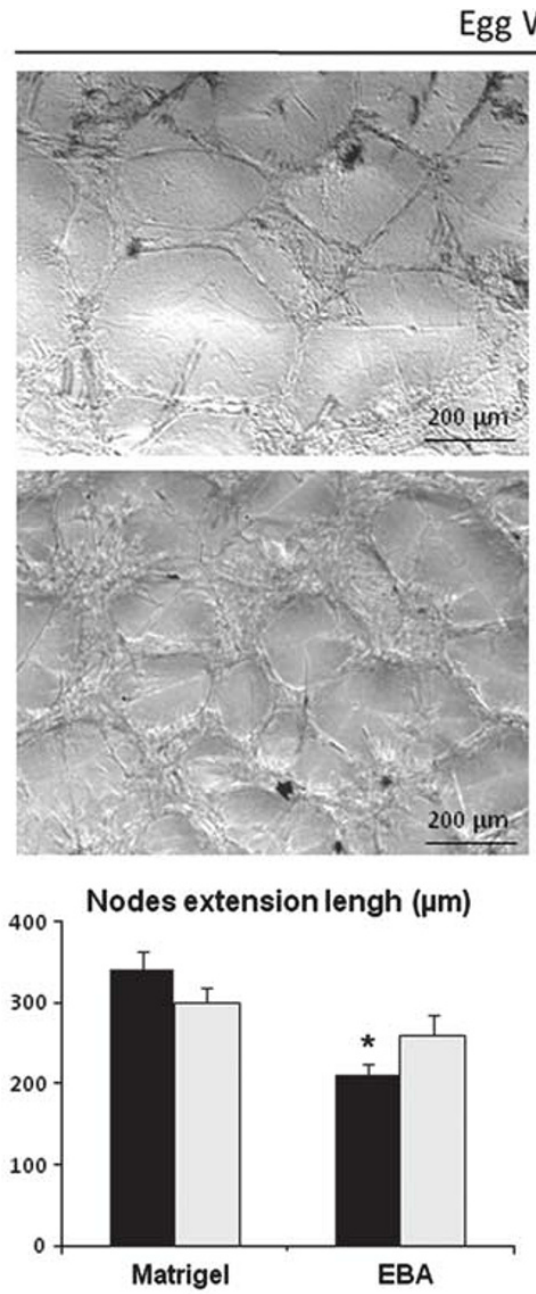
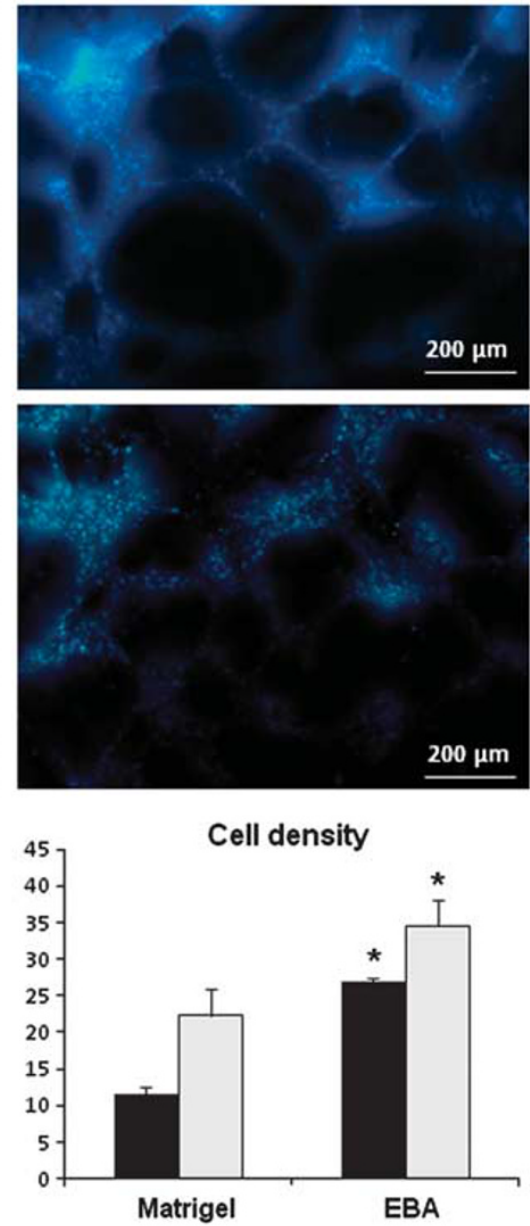

Figure 1 Endothelial cell growth and organization in egg white matrix versus Matrigel. Human umbilical vein endothelial cells (HUVECs) and rat aortic endothelial cells (RAECs) were seeded at $5 \times 10^{4}$ cells per well in a 24-well plate coated with Matrigel or egg white. Cell nuclei were labeled using Hoechst 33258 and photographed $48 \mathrm{~h}$ after seeding (magnification $\times 50$ ). Data are the means (s.e.m.) of three separate experiments $\left({ }^{*} P<0.05\right.$ vs Matrigel). 
network for RAECs as compared with that of HUVECs. If HUVEC and RAEC network characteristics were closely similar for a given matrix, cell nodes and cell density appeared significantly higher in egg white than in Matrigel.

HUVECs and RAECs organized themselves as a network with a similar kinetic. At $18 \mathrm{~h}$ after cell seeding, both cell types were adherent and the early stage of the cellular network could be observed (Figure 2). After $30 \mathrm{~h}$, the cellular network was clearly visible and was stable after 48 to $60 \mathrm{~h}$. Light or fluorescent microscopy showed obvious cell-specific network differences. HUVEC network was more regular looking like a 'grid' whereas RAEC network was more randomly disposed and composed of large cell nodes linked by cellular filaments of various length and thickness spreading in all directions.

\section{Network Specificity Depending on Multiple Cell Types in Coculture}

When RAECs and RASMCs were simultaneously seeded in egg white matrix, the resulting cellular network was much more spread in a larger area over $30 \mathrm{~mm}^{2}$ (Figure 3a). For this coculture, three-dimensional cell organization could slightly change until the end of the culture and cell network could be maintained for up to 18 days without significant cell death.

Increased magnification (Figures $3 \mathrm{~b}$ and $\mathrm{c}$ ) revealed $\mathrm{a}$ network composed of a greater number of cell nodes, and the cell fibers connecting the nodes appeared of bigger diameter (70 vs $26 \mu \mathrm{m}$ for RAECs only). The $\alpha$-SMA staining showed a peripheral location of RASMCs around apparent tubules composed of ECs (Figure 3e). These observations were sustained by 3D processed images from confocal $z$-stacking of Von Willebrand factor-labeled cells that revealed an internal cavity within the EC fiber mimicking the vascular formation (Figure 3f). The section view of this $3 \mathrm{D}$ construction revealed a cylindrical organization of cell nuclei and did not show any central cell into the fiber (Figure $3 \mathrm{~g}$ ).

Data quantification of networks composed of ECs or endothelial and smooth muscle cells (ECs + SMCs) showed that cocultured cells resulted in a significantly more structured
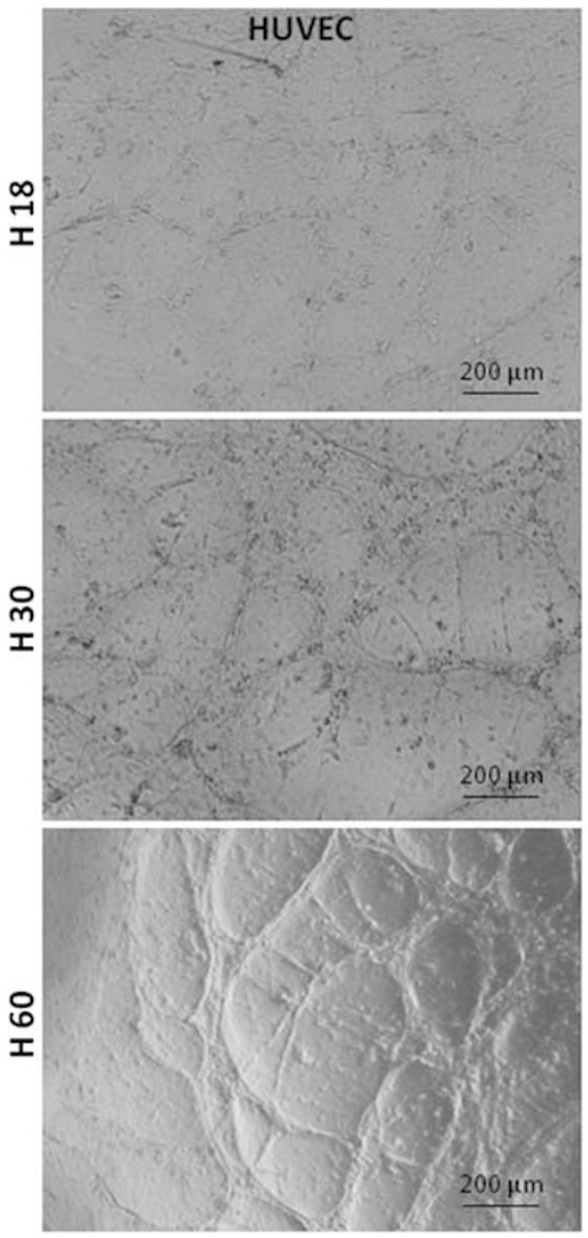
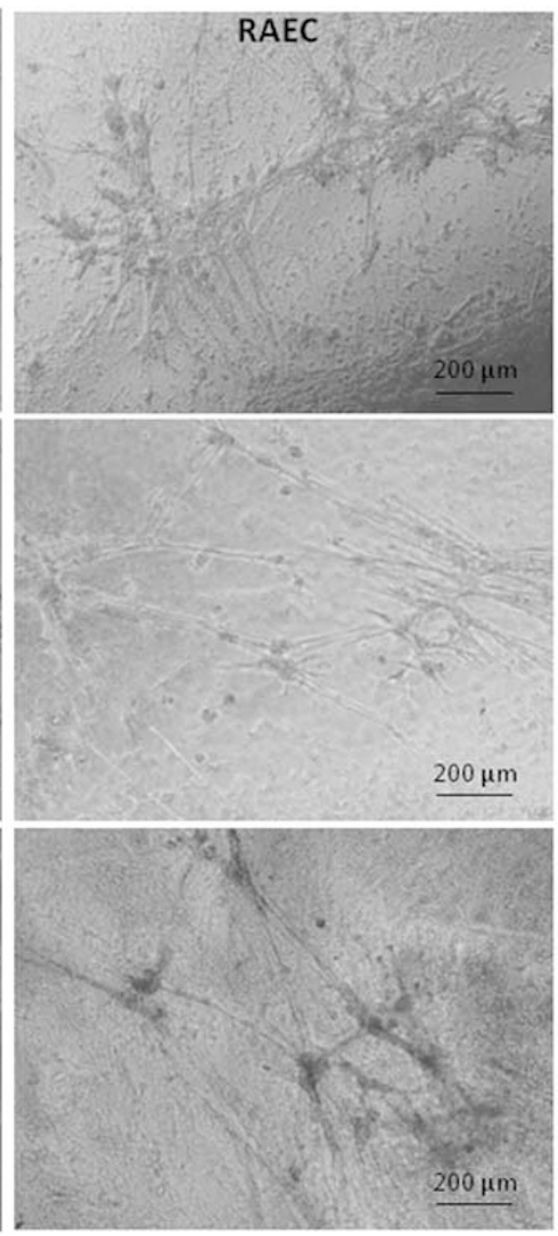
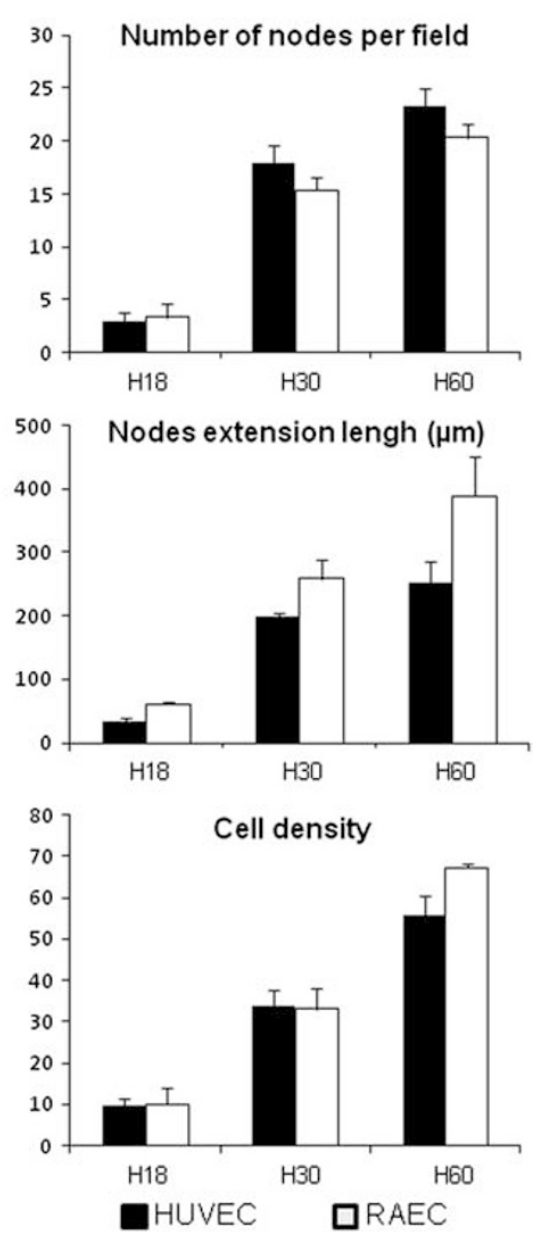

Figure 2 Network formation kinetic of endothelial cells in egg white matrix. Human umbilical vein endothelial cells (HUVECs) and rat aortic endothelial cells (RAECs) were seeded at $5 \times 10^{4}$ cells per well in a 24 -well plate coated with egg white. Cell nuclei were labeled using Hoechst 33258 after $12 \mathrm{~h}$ and cells were photographed 18,30 , and $60 \mathrm{~h}$ after seeding (magnification $\times 50$ ). Data are the means (s.e.m.) of three separate experiments. 


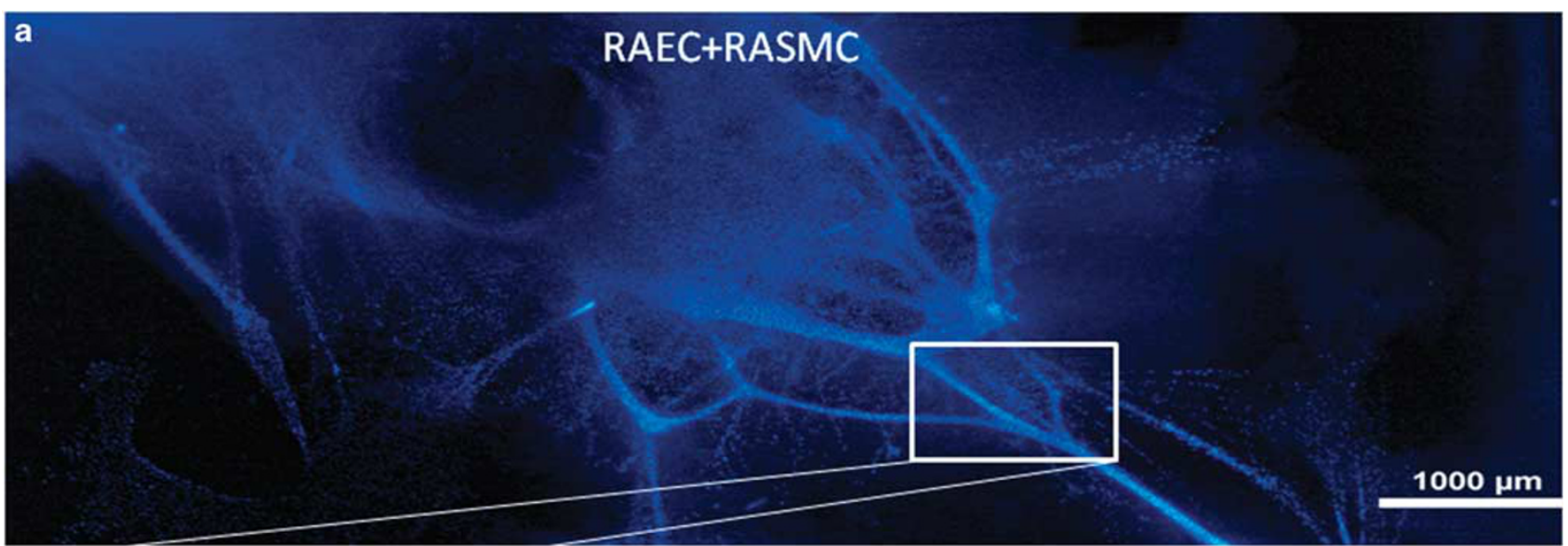

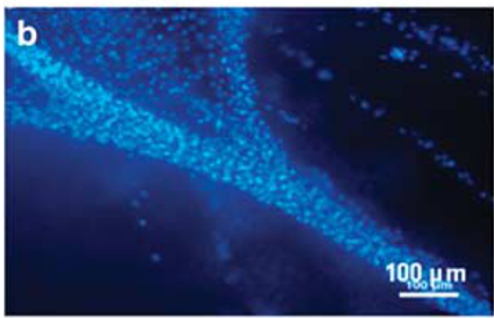

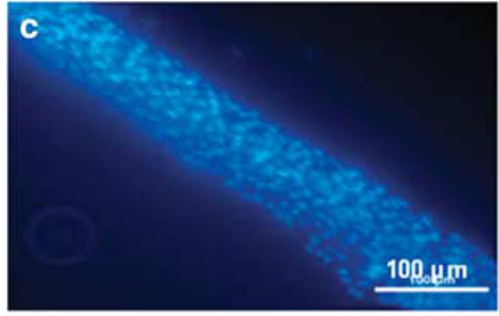

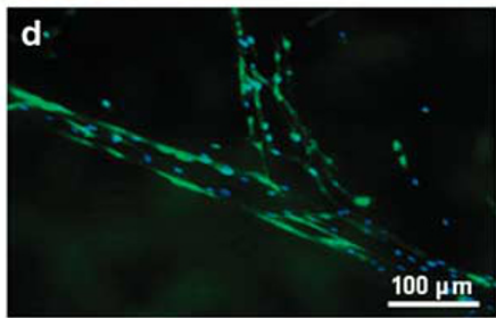

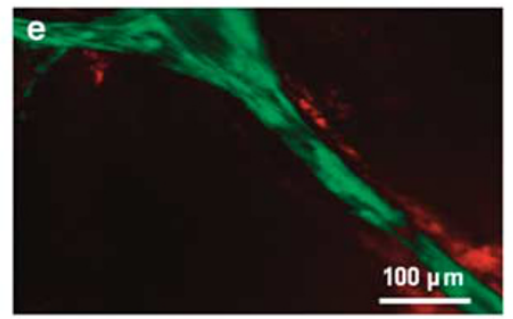

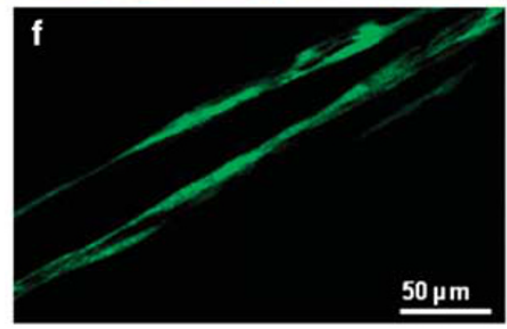

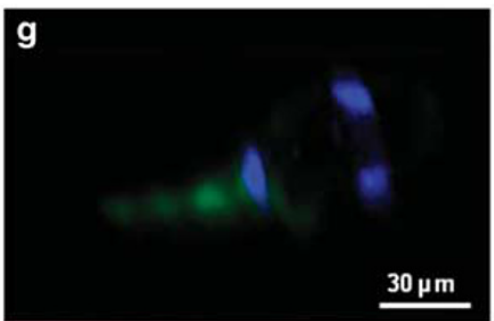

Figure 3 'Prevascular' organization of cocultured endothelial and smooth muscle cells in egg white matrix. Mixed (1:1) rat aortic endothelial cells (RAECs) and rat aortic smooth muscle cells (RASMCs) were respectively seeded at $2.5 \times 10^{4}$ cells per well in a 8 -well chamber slide or at $5 \times 10^{4}$ cells per well in a 24-well plate coated with egg white. Cell nuclei were labeled using Hoechst 33258 after $24 \mathrm{~h}$. Cell network was captured $72 \mathrm{~h}$ after seeding. (a) Using $\times 25$ magnification, the image composed of 18-assembled microscopic fields represents the whole cell network. (b) and (c) represent detailed view of the cell organization into a cylindrical structure at $\times 100$ and $\times 200$ magnifications, respectively. (d) Merged image of cells and Hoechst 33258. (e) Merged image of mixed (1:1) green fluorescent rat aortic endothelial cells (GRAECs) and RASMCs labeled with anti- $\alpha$-smooth muscle actin antibodies and phycoerytrin-coupled secondary antibodies. (f) Longitudinal view of the cylindrical structure formed by endothelial cells labeled with anti-von Willebrand factor antibodies. (g) Section view was obtained by confocal $z$-stacking and three-dimensional processed images of the cells forming cylindrical structure (magnification $\times 400$ ).

and spread network. Three approaches enabled us to clearly discriminate network formed by ECs alone or by a coculture of ECs + SMCs from human or rat origin (Figures $4 \mathrm{a}$ and $\mathrm{b}$, respectively). First, the number of cell nodes for a determined area showed that the network obtained from ECs was composed of $60 \%$ less cell nodes compared with the cocultured cells. Second, the cell extensions starting from nodes were 60 to $70 \%$ shorter in the case of ECs alone. And finally, the network density, defined as a ratio between cell labeling intensity and the area occupied by the cell network, was 50\% lower for ECs versus cocultured ones.

By using a vascular cell mix obtained by the enzymatic digestion of the thoracic aorta of a GFP-transgenic rat, we observed cells forming an alveolar structure (Figure 5a). The cells coming from the advantice formed a supportive tissue that stabilized, fed, and oriented EC and SMC organization in egg white (Figures $5 \mathrm{~b}$ and c). Cells organize themselves as concentrical circles with a very flat internal cell layer potentially mimicking vascular media surrounding intima, whole embedded in a matrix. This dense and spread cellular structure was stable after 4 days in the egg white. At day 5 , we seeded a small section of a rat ostesarcoma tumor obtained by the method previously described. ${ }^{19}$ At day 8 , the vascular cells have migrated toward ostesarcoma cells making the vascular network strongly directed toward the tumor (Figures $5 \mathrm{~d}$ and e).

\section{Aortic Ring Assay}

We compared cell sprouting from rat thoracic aorta sections in both Matrigel and egg white (Figures $6 \mathrm{a}-\mathrm{d}$ ). After 3 to 


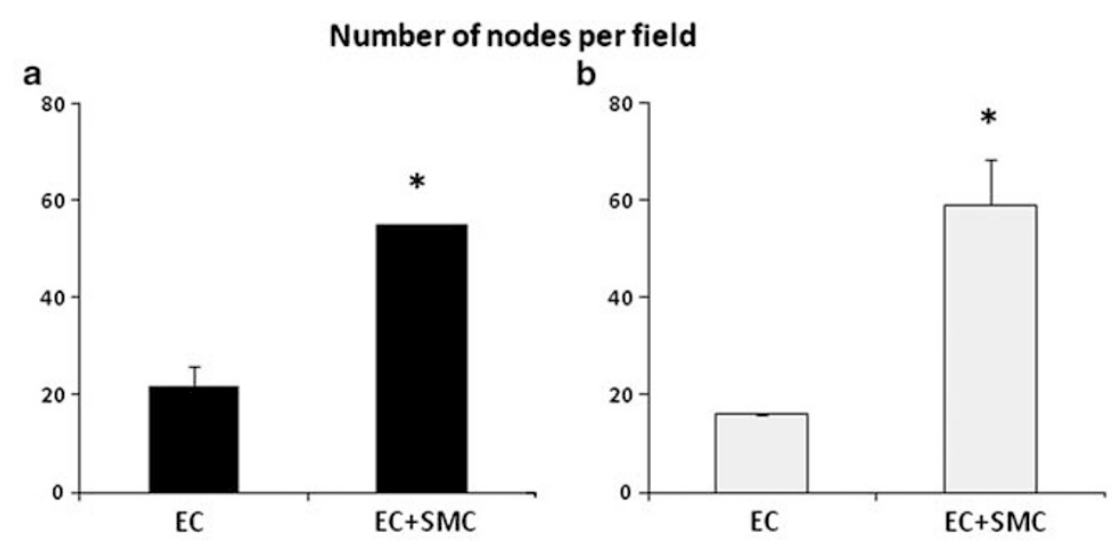

Nodes extension lengh $(\mu \mathrm{m})$
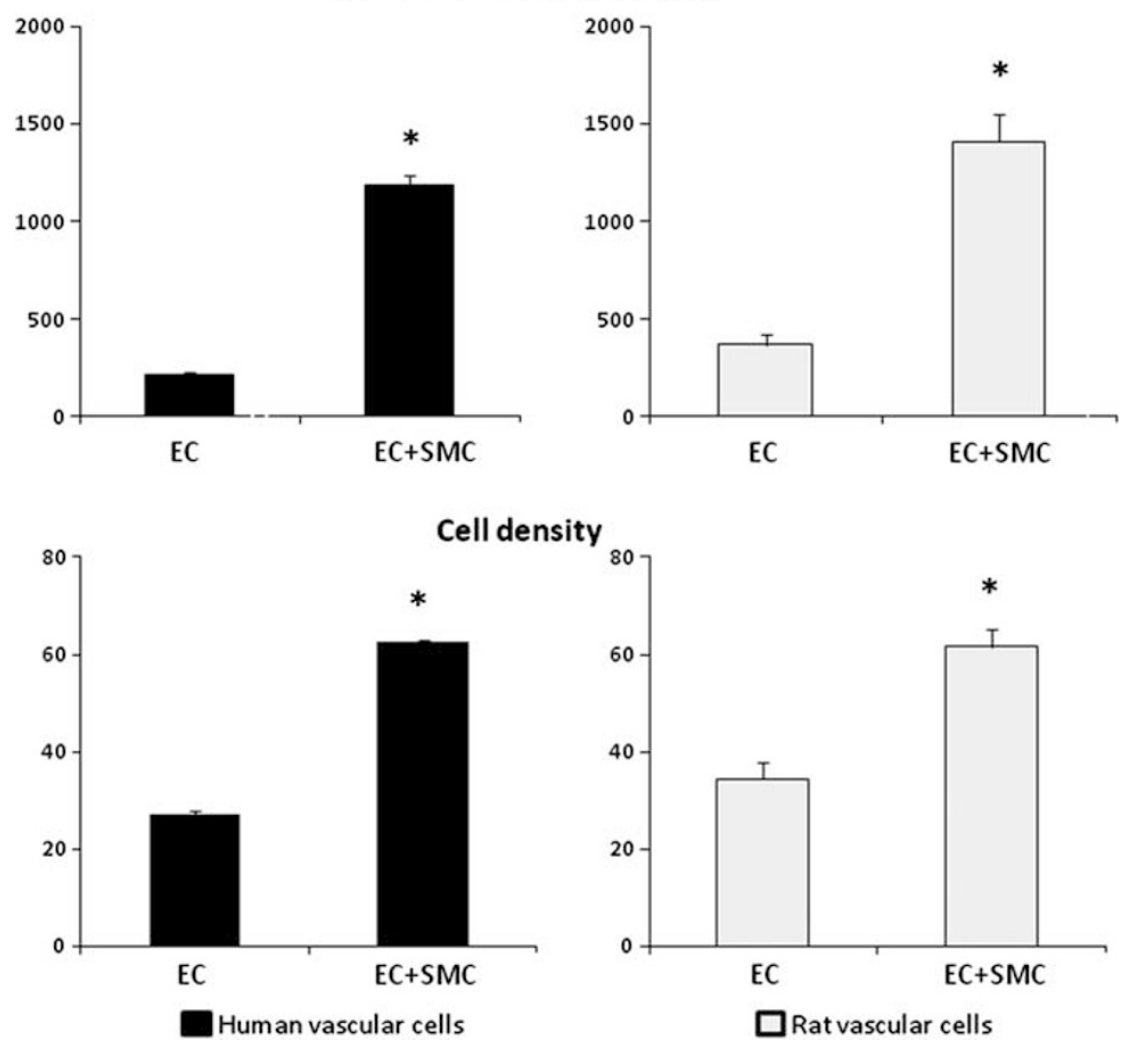

Figure 4 Data quantification of endothelial cells and smooth muscle cells forming network. Network parameter comparisons of endothelial cell monoculture $(E C)$ versus endothelial and smooth muscle cell coculture $(E C+S M C)$ from human (a) or rat origins (b). Human umbilical vein endothelial cells (HUVECs) and rat aortic endothelial cells (RAECs) were seeded at $5 \times 10^{4}$ cells per well in a 24-well plate coated with egg white. Mixed (1:1) HUVECs and human umbilical artery smooth muscle cells (HASMCs) or RAECs and rat aortic smooth muscle cells (RASMCs) were seeded at the same density. Cell network was captured 4 days after seeding with Hoechst 33258-labeled cells. Data are the means (s.e.m.) of three separate experiments $\left({ }^{*} P<0.05\right.$ vs EC).

5 days of culture, vascular cells sprouting from the aorta pieces could be observed through phase contrast microscopy. To assess that the first cells migrating from aorta wall were ECs , EC staining was performed by using anti-Von Willebrand factor antibodies (Figures 6e and f). However, cells observation using phase contrast microscopy was much more difficult in the egg white because it contain more fiber elements than Matrigel. Consequently, we seeded $2 \mathrm{~mm}$ long pieces of GFP-transgenic rat aortas in egg white. After 3 to 4 days, cells started to sprout from the vascular wall. Cells progressively organized themselves as a network and fully colonized the culture surface after 8 days. Similar to that observed with cell cultures, network development was higher in egg white than in Matrigel (Figure 6g). 

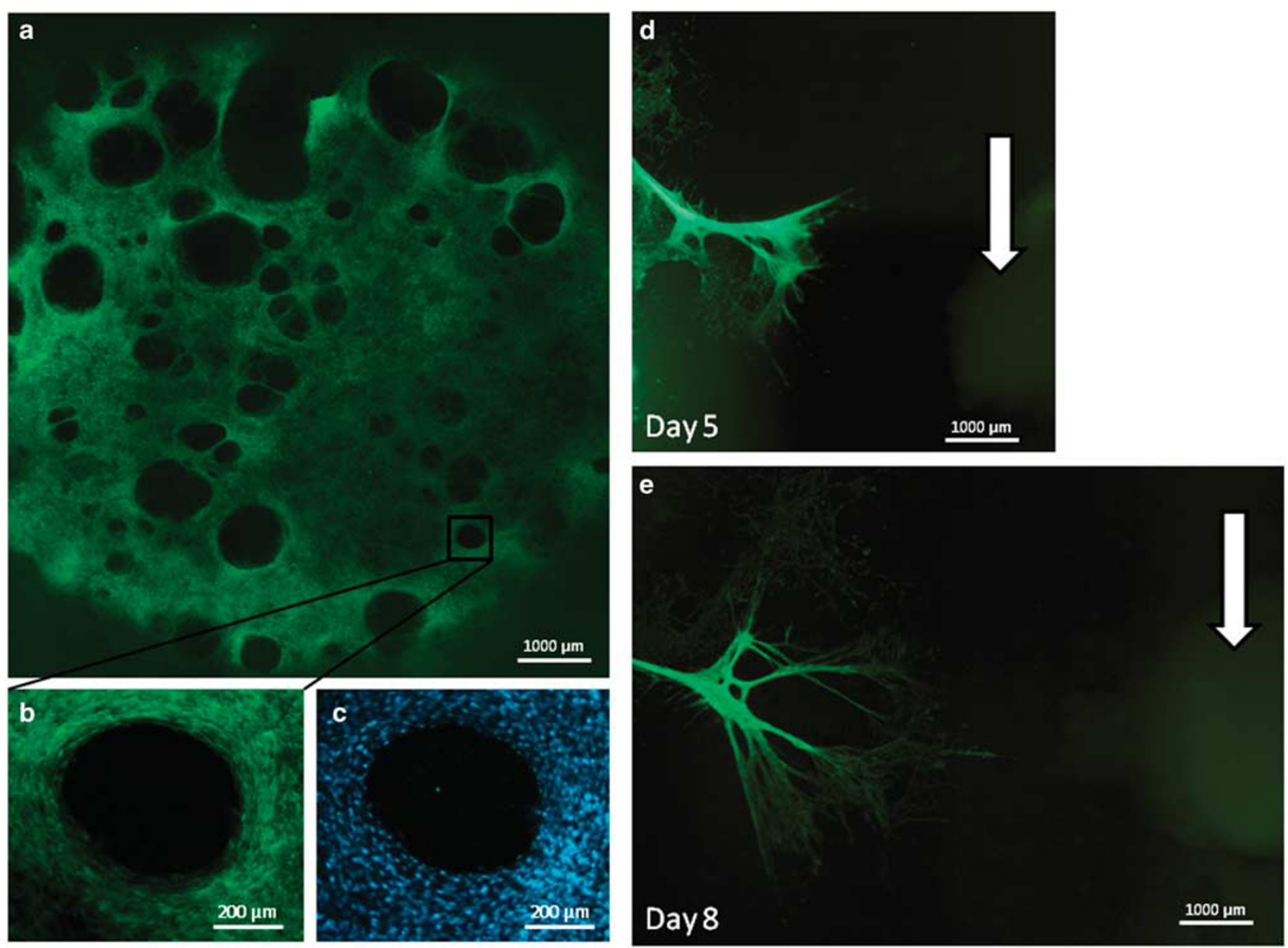

Figure 5 Network characteristics depending on cocultured cell types. (a) Alveolar structure formed by a vascular cell blend obtained from the thoracic aorta wall of a GFP-expressing rat. Using $\times 25 x$ magnification, the image composed of 24 -assembled microscopic fields represents the whole cell network. Cells were seeded at $5 \times 10^{4}$ cells per well in a 24-well plate coated with egg white. Cell network was captured 4 days after seeding and cell nuclei were labeled using Hoechst 33258 at $1 \mathrm{~h}$ before cell imaging. (b) Detailed view of GFP-expressing cells or (c) Hoechst 33258-labeled cells implicated in the network. (d, e) Coculture of a rat osteosarcoma tumor piece (white arrow) and prevascular network formed by rat vascular cell blend at $1 \mathrm{~h}$ and 3 days after osteosarcoma seeding, respectively.

Macroconfocal imaging enabled us to scan the entire colonized area by taking pictures of the cells every $16 \mu \mathrm{m}$ over $1 \mathrm{~mm}$ thick in $z$-stack and by building a 3D image of the network. After 8 days, cells could be spread in the egg matrix over $1 \mathrm{~mm}$ in depth and on a surface superior to $80 \mathrm{~mm}^{2}$ in a 24-well plate, whereas in Matrigel, cells colonized a smaller area and migrated from the aorta wall almost in a plane.

\section{Drug Testing in Egg White Matrix}

With the same experimental procedure using GFP-transgenic rat aortas seeded in egg white, we tested and compared with untreated control, epirubicin, sunitinib malate, and fingoli$\bmod$ (Figures $7 \mathrm{a}-\mathrm{d}$, respectively) on cells forming a vascular network. After 8 days, data analysis confirmed observations indicating that epirubicin or sunitinib malate totally inhibited vascular cell organization as a network (Figures $7 \mathrm{e}$ and f). These treatments did not induce a complete cell death, but cells just weakly spread in the aorta wall periphery. Fingolimod induced a network formation delay where cell spreading and organization was roughly comparable to that observed after 6 days without treatment.

\section{DISCUSSION}

Considering the results we obtained here with different cell types from various origins, egg white matrix appears to be a proper ECM substitute for angiogenesis assay. The egg white matrix used in our EBA was extracted from commercially available eggs and was compatible with cultured cells from human, mouse (data not shown), and rat origin. As it was described for mammary gland cells, ${ }^{15}$ vascular cells had a similar behavior in EBA than in other commonly used 3D matrix such as Matrigel. Regarding the principal cellular target of angiogenesis studies, ECs, their growth, chemotaxis, and spatial organization are closely similar in egg white or in 

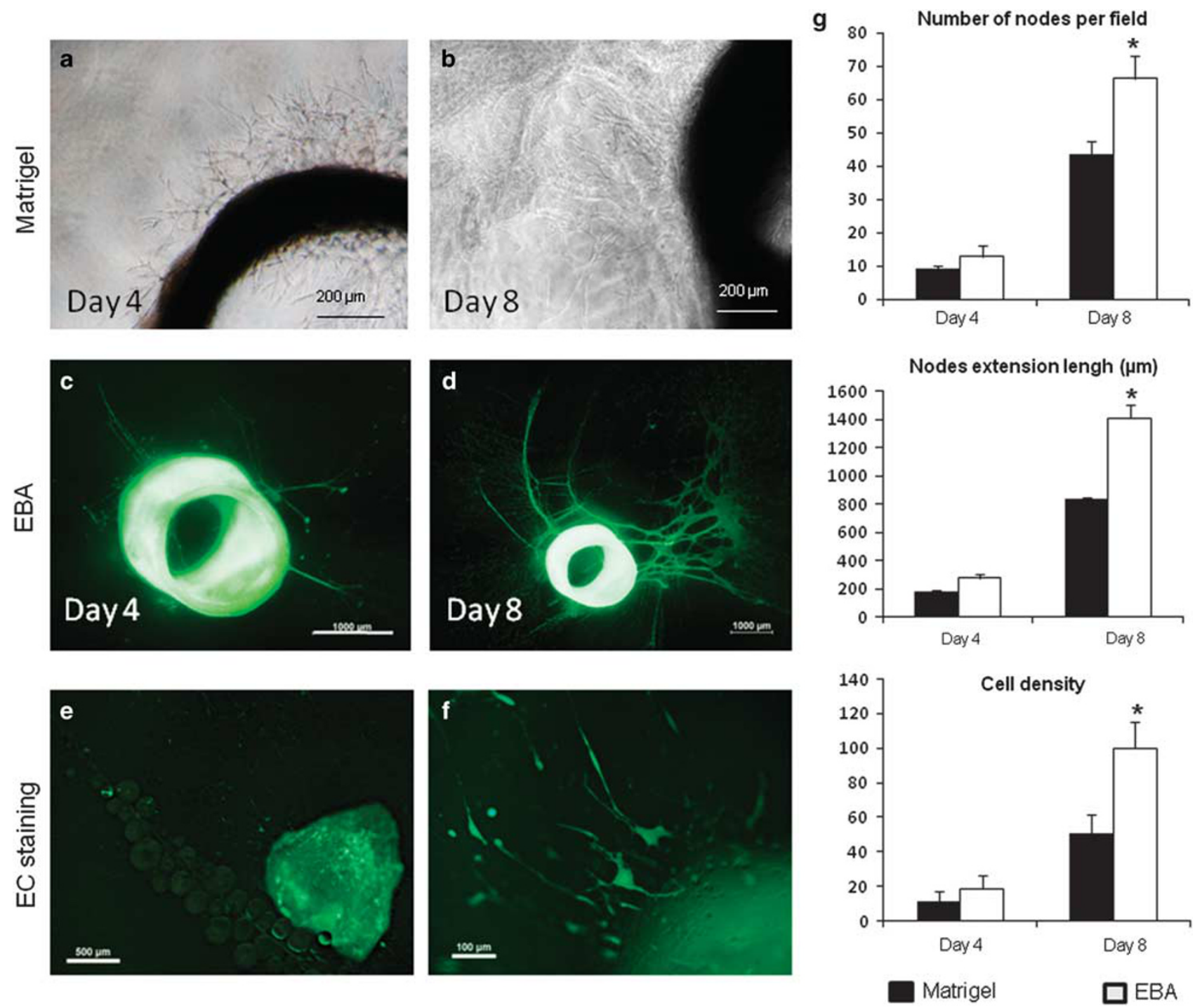

Figure 6 Aortic ring assay in egg white matrix vs Matrigel. (a, b) Rat aorta sections were seeded in 8-well chamber slide or 24-well plate coated with Matrigel. (c, d) Aorta sections from GFP-expressing rats were seeded similarly in plates coated with egg white. Images were captured 4 and 8 days after seeding. (e, f) Rat aorta sections seeded in 8-well chamber coated with egg white and labeled with anti-von Willebrand antibodies 3 days after seeding. (g) Data comparison after 4 and 8 days of cell network formed from aorta rings seeded in matrigel or egg white. Data are the means (s.e.m.) of three separate experiments $\left({ }^{*} P<0.05\right.$ vs Matrigel).

Matrigel. However, cell organization in EBA is slightly slower than in Matrigel. Cell network appeared clearly 2 days after seeding in EBA, whereas it can be visible after 1 day in Matrigel. In order to improve comparison, Matrigel was diluted $(1 / 2)$ to reach comparable network state 2 days after cell seeding. EBA enables endothelial and mural cell coculture and elicits network formation of these cells. We also cultured the whole variety of cells composing the vascular wall in EBA and then observed that cell interactions of this cell blend led to a specific pattern. In such conditions, our EBA showed that cell network formation was dramatically dependent on the single or multiple cell types seeded in such assay. As most of the multiple tyrosine kinase inhibitors in development or already in use in oncology target not only ECs but also mural cells through the platelet-derived growth factor (PDGF) or fibroblast growth factor (FGF) pathway, ${ }^{20}$ it could be of great interest to intensify the use of 3D angiogenic assays involving multiple cell types in order to properly address the question regarding the potential of these drugs. Similarly, we showed that we could obtain a 'prevascular' network formation from vascular cells and then a modification of this network induced by the addition of a tumor fragment. The ability to sequentially coculture vascular and tumor cells could potentially participate to distinguish antimitotic and antichemotactic effects of such treatments toward vascular cells. Through network adaptation in response to tumor stimuli, it also showed that vascular reorganization properties were preserved in EBA that represent key properties during tumor 

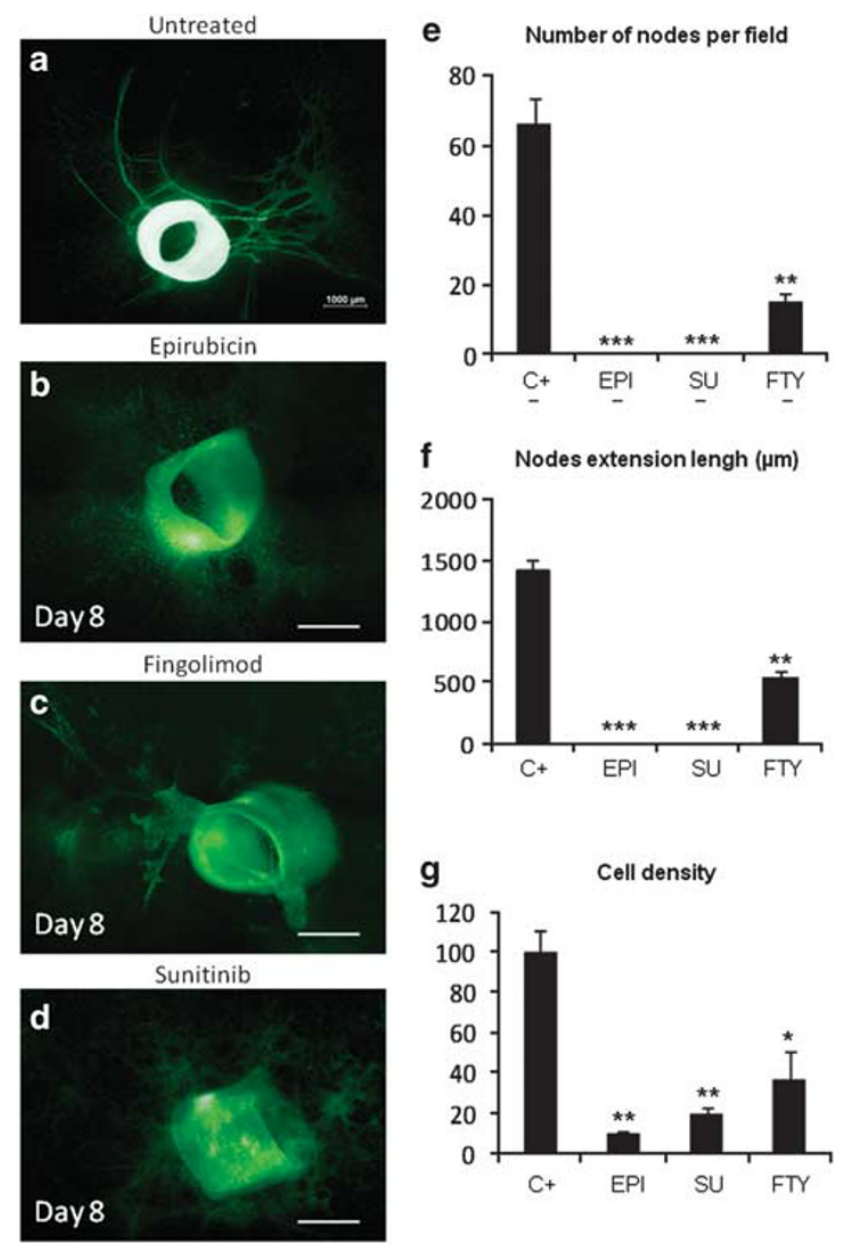

Figure 7 Anticancer drug testing in egg white matrix. (a) Aorta sections from GFP-expressing rats were seeded in 24-well plates coated with egg white. (b-d) At 2 days after seeding, aortic rings were treated with epirubicin (EPI, $0.1 \mathrm{mg} / \mathrm{ml})$, fingolimod (FTY, $100 \mathrm{nM})$, and sunitinib malate (SU, $1 \mu \mathrm{M})$, respectively. Image captures and data quantification $(\mathbf{e}-\mathbf{g})$ were performed 8 days after seeding. Each treatment was tested in triplicate ${ }^{*} P<0.05,{ }^{*} P<0.01$, and ${ }^{* * *} P<0.001$ vs untreated aortic ring, $(+)$.

angiogenesis or tissue graft. ${ }^{21-23}$ EBA appeared to be convenient to organotypic culture such as aortic ring assay that offers with its previously described properties a large potential in the field of angiogenesis assays. ${ }^{24,25} \mathrm{EBA}$ also provides a full compatibility with potential complementary experiments carried on the CAM model.

Numerous publications report that treatments combining two or three molecules have an improved anticancer effect versus single treatment. To assess the benefit of drug combination, it is necessary to be able to discriminate each drug effect. Most of the recently reported treatment combinations involve a cytotoxic agent. We tested epirubicin at low dose $(0.1 \mathrm{mg} / \mathrm{ml})$ to preserve cell viability and migration ability. We also tested sunitinib malate and fingolimod, and their benefits in combination on a rat breast cancer model have already been shown by us. ${ }^{26}$ According to our previous results reported on cell cultures and animal models, EBA experiments using aorta rings treated with a single drug resulted in specific inhibition of vascular network depending on the tested drug.

As Matrigel is now among the most popular angiogenesis assays, EBA could represent a very competitive alternative to such ECM, considering its cost. Moreover, Matrigel biological activity has already been described ${ }^{27,28}$ and strongly depends on its composition in growth factors. Experimentally, we faced variability in results in our laboratory that according to us could be because of variations in Matrigel composition depending on the provider or even between production lots of the same provider. On the contrary, with its availability and stability under freezing, egg white makes it possible to stock enough to work with a matrix of constant composition for a long period of time. In practice, it is easy to handle and EBA does not require any specific equipment. Regarding data analysis, egg white slightly increased opacity compared with Matrigel, making phase contrast observation of the cells more hazardous, although this problem can be easily addressed through cell staining with fluorescent probes. We successfully tested Hoechst 33258 and DAPI for nuclear staining as Mitotracker ${ }^{\mathrm{TM}}$ and PKH26 (Sigma) for cytosol and membrane labeling, respectively. As we already reported, ${ }^{26}$ such simple cell labeling enables data acquisition and network characterization, leading to objective antiangiogenic drug evaluation. As we demonstrated with aortic rings, the use of transgenic cells expressing a fluorescent protein such as GFP is of course the best choice for optimum simplicity and accuracy in microscopic observation. Protein expression can also be assessed with immunofluorescent labeling, taking into account that such experiments require that cells have to be fixed and rinsed several times that increases the risk of egg white matrix degradation. In fact, even after heating, egg white matrix is semi-solid like a gel and it keeps its plasticity. On one hand, these properties make it fragile, thus requiring it to be handled carefully. On the other hand, it implies for the cells forming a network to interconnect them with strong but also elastic junctions. This feature could more closely mimic mechanical constraint observed in physiological situations. In addition, we described in EBA a cell network developed in a deeper space (over $1 \mathrm{~mm}$ deep) than that we observed in Matrigel. If it represents a difficulty for standard or confocal imaging in which a deep field makes a proper focus hazardous, it suggests that cells are exposed to a gradient of oxygen and nutrient depending on their proximity to the egg white surface. This differential availability of oxygen and nutrient supply is also observed in various organs and is particularly critical for outgrowing tumors containing large necrotic areas.

Previous reports suggested that cell organization in 3D ECM substitute was depending on proteic micropatterns and proposed matrix content adaptation or modification. ${ }^{29,30}$ If it seems complicated to regulate spatial proteic micropattern in 
EBA, the new RAIN-droplet model could address this problem if defined vascular network had to be grown in EBA. In this model, preformed angiogenic network are produced by EC encapsulation in a specific hydrogel that consequently enhances cell growth and defines their starting orientation. ${ }^{31}$

In conclusion, as the use of animal models in drug discovery studies presents issues with feasibility and ethical concerns, ${ }^{32,33}$ we believe EBA represents a simple and relevant angiogenic assay. It offers a solution for most of the studies aiming at observing cell migration and organization in a $3 \mathrm{D}$ matrix and quantification of antiangiogenic drug properties in a cost-effective manner.

\section{ACKNOWLEDGMENTS}

We thank Claire Carion for her precious help in performing confocal imaging. We also thank Ignacio Anegon for his generous gift of GFPexpressing rats.

\section{DISCLOSURE/CONFLICT OF INTEREST}

The authors declare no conflict of interest.

1. Truskey GA. Endothelial cell vascular smooth muscle cell co-culture assay for high throughput screening assays for discovery of antiangiogenesis agents and other therapeutic molecules. Int J High Throughput Screen 2010;2010:171-181.

2. Pang Z, Niklason LE, Truskey GA. Porcine endothelial cells cocultured with smooth muscle cells became procoagulant in vitro. Tissue Eng Part A 2010;16:1835-1844.

3. Brown MA, Zhang L, Levering VW, et al. Human umbilical cord bloodderived endothelial cells reendothelialize vein grafts and prevent thrombosis. Arterioscler Thromb Vasc Biol 2010;30:2150-2155.

4. Wallace CS, Truskey GA. Direct-contact co-culture between smooth muscle and endothelial cells inhibits TNF-alpha-mediated endothelial cell activation. Am J Physiol Heart Circ Physiol 2010;299:H338-H346.

5. Derda R, Laromaine A, Mammoto A, et al. Paper-supported 3D cell culture for tissue-based bioassays. Proc Natl Acad Sci USA 2009;106: 18457-18462.

6. Derda R, Tang SK, Laromaine A, et al. Multizone paper platform for 3D cell cultures. PLoS One 2011;6:e18940.

7. Rae PC, Kelly RD, Egginton S, et al. Angiogenic potential of endothelial progenitor cells and embryonic stem cells. Vasc Cell 2011;3:11.

8. Chen Z, Htay A, Dos Santos W, et al. In vitro angiogenesis by human umbilical vein endothelial cells (HUVEC) induced by three-dimensional co-culture with glioblastoma cells. J Neurooncol 2009;92:121-128.

9. Holmes K, Chapman E, See V, et al. VEGF stimulates RCAN1.4 expression in endothelial cells via a pathway requiring $\mathrm{Ca} 2+/$ calcineurin and protein kinase C-delta. PLoS One 2010;5:e11435.

10. Dohle $E$, Fuchs $S$, Kolbe $M$, et al. Comparative study assessing effects of sonic hedgehog and VEGF in a human co-culture model for bone vascularisation strategies. Eur Cell Mater 2011;21:144-156.

11. Roberts OL, Holmes K, Muller J, et al. ERK5 is required for VEGFmediated survival and tubular morphogenesis of primary human microvascular endothelial cells. J Cell Sci 2010;123(Pt 18):3189-3200.
12. Mikirova NA, Casciari JJ, Riordan NH. Ascorbate inhibition of angiogenesis in aortic rings ex vivo and subcutaneous Matrigel plugs in vivo. J Angiogenes Res 2010;2:2.

13. Dohle DS, Pasa SD, Gustmann $S$, et al. Chick ex ovo culture and ex ovo CAM assay: how it really works. J Vis Exp 2009;33:1620.

14. Norrby K. In vivo models of angiogenesis. J Cell Mol Med 2006;10: 588-612.

15. Kaipparettu BA, Kuiatse I, Tak-Yee Chan B, et al. Novel egg white-based 3-D cell culture system. Biotechniques 2008;45:165-168, 170-161.

16. Crampton SP, Davis J, Hughes CC. Isolation of human umbilical vein endothelial cells (HUVEC). J Vis Exp 2007;3:183.

17. Kwan HY, Huang Y, Yao X. Store-operated calcium entry in vascular endothelial cells is inhibited by cGMP via a protein kinase G-dependent mechanism. J Biol Chem 2000;275:6758-6763.

18. Sachinidis A, Flesch M, Ko $Y$, et al. Thromboxane A2 and vascular smooth muscle cell proliferation. Hypertension 1995;26:771-780.

19. Dutour A, Rabinovich-Chable H, Kaletta $\mathrm{C}$, et al. Is troponin I gene therapy effective for osteosarcoma treatment? Study on a human-like orthotopic rat model. Anticancer Res 2004;24:3977-3982.

20. Cook KM, Figg WD. Angiogenesis inhibitors: current strategies and future prospects. CA Cancer J Clin 2010;60:222-243.

21. Bohman S, Matsumoto $\mathrm{T}$, Suh $\mathrm{K}$, et al. Proteomic analysis of vascular endothelial growth factor-induced endothelial cell differentiation reveals a role for chloride intracellular channel 4 (CLIC4) in tubular morphogenesis. J Biol Chem 2005;280:42397-42404.

22. Chang CC, Krishnan L, Nunes SS, et al. Determinants of microvascular network topologies in implanted neovasculatures. Arterioscler Thromb Vasc Biol 2012;32:5-14.

23. Secomb TW, Dewhirst MW, Pries AR. Structural adaptation of normal and tumour vascular networks. Basic Clin Pharmacol Toxicol 2012;110:63-69.

24. Nicosia RF, Zorzi $\mathrm{P}$, Ligresti $\mathrm{G}$, et al. Paracrine regulation of angiogenesis by different cell types in the aorta ring model. Int J Dev Biol 2011;55:447-453.

25. Goodwin AM. In vitro assays of angiogenesis for assessment of angiogenic and anti-angiogenic agents. Microvasc Res 2007;74: 172-183.

26. Mousseau Y, Mollard S, Faucher-Durand K, et al. Fingolimod potentiates the effects of sunitinib malate in a rat breast cancer model. Breast Cancer Res Treat 2011;134:31-40.

27. Kleinman HK, Martin GR. Matrigel: basement membrane matrix with biological activity. Semin Cancer Biol 2005;15:378-386.

28. Hughes CS, Postovit LM, Lajoie GA. Matrigel: a complex protein mixture required for optimal growth of cell culture. Proteomics 2010;10:1886-1890.

29. Sodunke TR, Turner KK, Caldwell SA, et al. Micropatterns of Matrigel for three-dimensional epithelial cultures. Biomaterials 2007;28: 4006-4016.

30. Cronin KJ, Messina A, Thompson EW, et al. The role of biological extracellular matrix scaffolds in vascularized three-dimensional tissue growth in vivo. J Biomed Mater Res B Appl Biomater 2007;82:122-128.

31. Zeitlin BD, Dong Z, Nor JE. RAIN-Droplet: a novel 3D in vitro angiogenesis model. Lab Invest 2012;92:988-998.

32. Maltman DJ, Przyborski SA. Developments in three-dimensional cell culture technology aimed at improving the accuracy of in vitro analyses. Biochem Soc Trans 2010;38:1072-1075.

33. Elliott NT, Yuan F. A review of three-dimensional in vitro tissue models for drug discovery and transport studies. J Pharm Sci 2011;100:59-74. 